\title{
Artículos
}

\section{Impacto ecológico, social y económico de fincas certificadas en buenas}

prácticas agrícolas y comercio justo*

Cómo citar este artículo: Caviedes Rubio, D., y Olaya Amaya, A. (2020). Impacto ecológico, social y económico de fincas certificadas en buenas prácticas agrícolas y comercio justo. Cuadernos de Desarrollo Rural, i7. https://doi.org/I0.III44/Javeriana.cdri7.iese

Diego Iván Caviedes Rubio ${ }^{\text {a }}$

Universidad Cooperativa de Colombia, Colombia

diegoivancaviedes@gmail.com

ORCID: https://orcid.org/0000-0002-6IOI-9II4

Alfredo Olaya Amaya

Universidad Surcolombiana, Colombia

ORCID: https://orcid.org/0000-0002-7080-I394

DOI: https://doi.org/I0.III44/Javeriana.cdrı7.iese

Recibido: 15 Noviembre 2018 I Aceptado: 15 Noviembre 2019 I Publicación: 22 Octubre 2020

\section{Resumen:}

Se realizó una revisión de diversos estudios enfocados para establecer el impacto ambiental generado en las fincas productoras de café certificadas con diferentes sellos de buenas prácticas agrícolas y socioeconómicas en las diversas regiones productoras del grano. De esta revisión se establecieron los impactos de mayor persistencia, así como los de mayor singularidad en todo el mundo.

Palabras clave: buenas prácticas agrícolas, certificación agrícola, impacto ambiental.

Ecological, Social and Economic Impact of Farms Certified in Good Agricultural Practices and Fair Trade

a Autor de correspondencia. Correo electrónico: diegoivancaviedes@gmail.com 


\section{Abstract:}

A review was conducted on some focused studies in order to determine the environmental impact caused by coffee-producing farms certified by different Good Agricultural and Socio-Economic Practices systems in different coffee-growing regions. This review shows the most persistent impacts as well as the most particular ones worldwide.

Keywords: good agricultural practices, agricultural certification, environmental impact.

\section{Introducción}

Luego de diversas crisis en el mercado cafetero y ante las escasas oportunidades para los pequeños productores de incrementar su participación en los beneficios del comercio internacional de este producto, surgió la alternativa de apoyar la creación de vínculos de los pequeños agricultores a los mercados de cafés especiales. Incluidos los destinados a la certificación de comercio justo y, el café orgánico, con lo que se ha buscado mejorar las perspectivas de los pequeños productores en el corto y largo plazo (Donovan y Poole, 20I4).

El mercado de los cafés especiales ha evidenciado un rápido crecimiento por su alta demanda. Aunque para acceder a estos mercados los agricultores han tenido que cumplir una serie de requisitos estrictos, que generalmente, conducen a la certificación por parte de una entidad u órgano acreditador al cual los productores solicitan inspecciones para verificar las condiciones de producción. Dicha solicitud, la hacen de forma independiente. La certificación de fincas de café como orgánico, comercio justo y/o de sombra, es una alternativa de protección a la biodiversidad y promueve salarios dignos para los agricultores. Si se cumplen los criterios de la agencia de certificación, se autoriza un sello de aprobación en los envases. De ese modo, se garantiza a los consumidores que los criterios específicos del programa de certificación, se cumplen en la granja donde se cultivan productos (Milder, 20I4).

La diferenciación en el mercado, presupone a una empresa o consumidor para una demanda sustancial de productos sostenibles. Esta demanda podría reflejarse en la disposición de pagar precios más altos para los productos certificados como sostenibles, o en las expectativas de que ciertas líneas de productos se derivan sólo de fuentes sostenibles. Los productores pueden beneficiarse de precios más altos para los productos certificados, lo cual da lugar a un incentivo económico para que estos adopten prácticas sostenibles (Ferraro et al., 2005) y aseguren el acceso a un mercado. Pues de otro modo podrían quedar excluidos (Tscharntke et al., 2015).

Se siguió una línea de investigación sobre la evaluación del impacto socioeconómico y ecológico bajo las condiciones de los productores certificados. A pesar de que los organismos de certificación generaron numerosas evaluaciones de impacto real de sus programas a corto y largo plazo, los resultados fueron contradictorios y todavía se hace necesaria una investigación de mayor calidad en diferentes escenarios (Takahashi y Todo, 20I3; Trimarchi, 2015).

Un estudio de revisión desarrollado por Blackman y Rivera (2010), analiza diferentes investigaciones sobre el impacto ambiental, generado por las certificaciones de sostenibilidad condiciones laborales y comercio justos, en la producción de varios productos agrícolas. Para el caso del café se reportaron 26 investigaciones de las cuales solo 6 presentan una estructura razonable y verificable para el estudio de impacto. Tales estudios evidenciaron que, a nivel de fincas certificadas, no se proporcionan pruebas convincentes de que la certificación refleje impactos positivos a nivel socioeconómico y ambiental (Trimarchi, 20I5).

Estas investigaciones evalúan comparativamente los impactos de la producción certificada respecto a la no certificada. No existen evaluaciones del impacto basadas en el antes y después de la certificación. Arnould et al. (2009) y Bolwig et al. (2009), indican que la certificación tiene beneficios socioeconómicos significativos, Blackman y Naranjo (2010) concluyen que la certificación presenta impactos ambientales importantes, mientras que para 
Sáenz y Zuñiga (2008), Fort y Ruben (2008), Lyngbaek et al. (200I) y Rivera et al (2006) la certificación no evidencia ningún impacto relevante. Se observa un mayor grado de evaluación sobre la certificación FLO, comercio justo y muy poca sobre otros programas igualmente importantes (Krasnozhon et al., 2015).

De acuerdo con Philpott et al. (2007) y Barham y Weber (2012) los estudios sobre resultados ambientales de certificación que emplean muestras aleatorias representativas son escasos. Mientras otros, son muy optimistas en sus resultados debido a la falta de control del sesgo de autoselección (Blackman y Naranjo, 20r2), los cuales se enfocan en reforestación o cultivos con sombrío (Haggar et al., 2015; Hardt et al., 2015; Rueda et al., 2015), calidad del suelo, uso de agroquímicos, diversidad y abundancia de aves (Martínez-Sánchez, 2008; MartínezTorres, 2008; Jaffee, 2008). Así mismo, es evidente que los criterios ambientales que tienen en cuenta los programas de certificación difieren entre sí, lo que afecta su comparación (Ponte, 2004; Van Rijsbergen et al., 2016). Finalmente, estudios realizados por Raynolds et al. (2007), indican que la vulnerabilidad de estas iniciativas a las presiones del mercado, pone de relieve la necesidad de una regulación privada en conjunto con la regulación pública, para trabajar en la mejora de la sostenibilidad social y ambiental.

\section{Metodología}

Basados en las indicaciones referidas en el Cocbrane Handbook for Systematic Reviews of Interventions (Higgins y Green, 20II) se definió la metodología para este proceso de obtención, selección y análisis de documentación.

Inicialmente se estableció el alcance de la pregunta de investigación, definiendo esta como el grado de conocimiento en la literatura sobre el impacto ambiental que ha generado la adopción de programas de certificación en buenas prácticas ecológicas, económicas y sociales en las fincas cafeteras de los principales países productores del grano.

Se realizó una revisión documental sobre los países con mayor productividad cafetera según la International Coffee Organization [ICO] (2017) y se identificó por continente (Asia, África, Centroamérica - México y Suramérica) las naciones con mayor áreas cultivadas con café bajo los principales sellos de certificación (Bird-Friendly, Smithsonian Migratory Bird Center, Rainforest Alliance [RAS], Fairtrade Labelling Organizations International [FLO], UTZ Certified y Orgánico) y según la especie predominante.

Los artículos empleados en este estudio se obtuvieron luego de la revisión en las siguientes bases de datos: Scopus, Science Direct, Springer Link, JSTOR, Web of Science y Scielo. En total se revisaron 106 artículos de los cuales se seleccionaron 78 documentos que cumplieron con los siguientes criterios:

- Estar publicados en revistas de divulgación científica de reconocida trayectoria y relevancia académica

- Estar relacionados con programas de certificación en BPA adoptadas en el sector caficultor de cada continente.

- Que el documento indique claramente el programa de certificación al que se refieren, los cuales deben ser (RAS, FLO, UTZ, Bird-Friendly y Orgánico).

- Investigaciones realizadas en países líderes en producción caficultora encada continente.

- Estar publicado entre los años 2000 y 2018

- Estar escritos en idioma ingles

La información obtenida se registró en una tabla en donde se clasificaron los impactos según su naturaleza en las categorías: ecológico, económico y social. Luego se homogeneizaron los impactos por su similaridad y la definición dada por los autores, identificando así los impactos que en cada continente se presentan con mayor frecuencia. 


\section{Principales productores de café certificado en el mundo}

Según datos publicados por la International Coffee Organization (2017) en el año 2016 la producción global de café fue de aproximadamente 148 millones de sacos de $60 \mathrm{Kg}$. El 45,3\% de la producción mundial se genera en Suramérica, donde Brasil y Colombia, primer y tercer productor del planeta respectivamente, entre los dos generan el 93,2 \% de la producción en el continente. Por su parte, Asia aporta el 31,8 \% del café mundial, en la que países como Vietnam (segundo productor mundial), Indonesia e India contribuyen con el 94,6 \% del café asiático.

El continente africano cuyos principales productores son Etiopia y Uganda produjo en el año 2016 aproximadamente $\mathrm{II}, 3$ \% del grano que se consume en el mundo, una cantidad muy similar a la de Centroamérica y México, donde países como Honduras, Guatemala y Nicaragua son los principales productores de la región. Finalmente cabe destacar a Papúa, Nueva Guinea como el principal productor de café en Oceanía con una producción de 712.000 sacos en 2016 que representa el $0,5 \%$ del café mundial.

Para el año 20I4, se registraron un aproximado de 4,I millones de hectáreas certificadas bajo uno de los 5 principales sellos de cumplimiento de estándares de sostenibilidad voluntarios (FIBL y IFOAM, 2016). Estos sellos hacen referencia a los programas de certificación: Bird-Friendly (Smithsonian Migratory Bird Center, 2017), Rainforest Alliance (RAS), Fairtrade Labelling Organizations International (FLO), UTZ Certified y el ${ }_{4} \mathrm{C}$ Code of Conduct, aunque cabe aclarar que esta no es el área real de plantaciones certificadas, ya que una misma hectárea de café puede estar certificada bajo varios programas. En la tabla i se pueden observar las áreas certificadas en los tres principales continentes productores de café bajo cuatro de los principales programas de certificación.

TABLA 1.

Áreas certificadas en Cada Continente PRoductor de café

\begin{tabular}{lcccc}
\hline & $\begin{array}{c}\text { Bird-Friendly } \\
\text { ha }\end{array}$ & UTZ ha & RAS ha & FLO ha \\
\hline Latinoamérica & 5065 & 338.367 & 273.000 & 829.200 \\
Asia & 48 & 120.237 & 60.300 & 70.020 \\
África & 626 & 90.426 & 31.500 & 206.300
\end{tabular}

Fuentes: UTZ (2015); Smithsonian Migratory Bird Center (2017); Milder y Newson (2015); Fairtrade International (2015)

\section{Impacto ecológico, social y económico de fincas certificadas}

A continuación se expondrán algunas características de la producción cafetera en Asia, África, Centroamérica y Suramérica y los impactos ambientales desde el punto de vista ecológico y socioeconómico generados por la adopción de diferentes programas de certificación en estos continentes. 


\section{Asia}

La producción de café en el continente asiático genera aproximadamente el 31,6 \% del café mundial (ICO, 20I7) y se obtiene principalmente en 9 países: Vietnam, Indonesia, India, Filipinas, Laos, Tailandia, Yemen, Camboya y China con predominio en las plantaciones de la especie Robusta, cuyas parcelas certificadas ligeramente exceden una hectárea (UTZ, 2015; Fairtrade International, 2015).

Luego de revisar diferentes documentos publicados sobre los efectos de la certificación de cultivos de café en territorio asiático, se expone en la tabla 2 los principales impactos ecológicos, sociales y económicos que predominan en los reportes de los estudios desarrollados de Vietnam, Indonesia, China e India. Países donde se han realizado un mayor número de investigaciones.

TABLA 2.

Principales impactos generados por la Certificación de Plantaciones de CAfÉ en Asia

\begin{tabular}{|c|c|}
\hline Impactos ecológicos & Impactos económicos \\
\hline $\begin{array}{l}\text { - Reducción del uso de plaguicidas y } \\
\text { abonos sintéticos } \\
\text { - Incremento de medidas de ahorro del } \\
\text { agua } \\
\text { - Aumento del reciclaje de materiales }\end{array}$ & $\begin{array}{l}\text { - Aumenta la estabilidad en los precios } \\
\text { - Aumento de la capacidad de } \\
\text { asociación } \\
\text { - Ingresos netos más altos } \\
\text { - Desarrollo de redes regionales de } \\
\text { productores }\end{array}$ \\
\hline Impactos Sociales & $\begin{array}{l}\text { - Incapacidad de cooperativas para } \\
\text { asegurar el apoyo a caficultores }\end{array}$ \\
\hline $\begin{array}{l}\text { - Aumento de la educación y } \\
\text { capacitación en producción cafetera }\end{array}$ & $\begin{array}{l}\text { - Limitación en el empoderamiento } \\
\text { económico y político }\end{array}$ \\
\hline
\end{tabular}

Fuente: elaboración propia

La certificación ofrece un vehículo para el reconocimiento del mercado de buenas prácticas, disminuye significativamente el uso de plaguicidas y abonos sintéticos promoviendo el uso de abonos orgánicos a partir del reciclaje de diversos materiales residuales generados en la finca. Todas las certificaciones incluyen criterios a favor de la protección de las fuentes locales de agua, por lo que se hace evidente la adopción de medidas de ahorro del agua (COSA, 2013, Milder y Newson, 2015).

La certificación también apoya otros servicios de los ecosistemas de las áreas de producción, tales como el almacenamiento de carbono, la purificación y regulación del flujo de agua (Tscharntke et al., 20II). Los estudios realizados por Kessler et al., (2012) en Indonesia, sugieren que 1,6 ha de agrobosque manejados de forma óptima puede capturar el carbono equivalente a cómo iha de bosque natural lo haría. Pero también se observó que no hay un vínculo estrecho entre el almacenamiento de carbono y la biodiversidad, ya que en estos agroecosistemas forestales se encontró una baja biodiversidad comparada con el bosque nativo aledaño. 
El principal impacto social registrado en Asia, es la reducción de la mano de obra infantil en la producción cafetera, vale la pena aclarar que la mayoría de pequeños productores lo consideran una actividad familiar en la que se incluyen todos sus miembros, lo que hace de este un impacto ambiguo (Auriol y Schilizzi, 2015). Por otro lado Baland y Duprez (2009), indican que cuando la obtención de una etiqueta es relativamente fácil, su impacto se reduce significativamente, debido al fenómeno de desplazamiento que se origina en las labores de la finca; ya que los trabajadores adultos reemplazan a los niños en las plantaciones certificadas y los niños sustituyen a los adultos en el sector doméstico. Burlando de cierta manera este criterio de certificación.

La producción de café bajo las normas ambientales y sociales de los programas de certificación en Vietnam e Indonesia, permiten a los agricultores beneficiarse de precios más estables, a los miembros de las cooperativas constituidas por pequeños productores propietarios de fincas certificadas, les garantiza un ingreso neto más alto (Chi et al., 20I5), lo cual permite contrarrestar las fluctuaciones de los precios del café y sus efectos nocivos sobre los medios de vida. Además los impulsa hacia un sistema asociativo más descentralizado y participativo (Meyfroidt et al., 2013).

En Indonesia se ha hecho notorio el aumento de empresas comerciales multinacionales en las zonas productoras de café (Neilson, 2008), lo que en la mayoría de los casos, ha permitido a los caficultores aprovechar su apoyo técnico y programas de formación o capacitación, en conjunto con los programas de certificación (Fairtrade International, 2015; Zapata y Nauges, 2016). Sin embargo, también se ha presentado incapacidad por parte de las cooperativas para asegurar el apoyo a los agricultores, debido a que no proporcionan los mismos servicios de mercado tradicionales, como el acceso sin problemas a los procedimientos de comercialización dentro de los ciclos de mercado tradicionales de crédito (Neilson, 2008).

En Vietnam Auld (2010), identificó un impacto que se podría generalizar en toda Asia e incluso otros continentes. Tal impacto es derivado del auge del café certificado, en relación con la demanda y los precios por su comercialización, pues han puesto en evidencia la limitación de los avances en el empoderamiento tanto económico como político de los productores derivado del poder que tienen los comerciantes y tostadores de café en estos sectores.

\section{África}

Los principales productores de café en África: Etiopía, Uganda, Costa de Marfil, Kenia, Camerún, Tanzania, Madagascar y Ghana producen el II,5\% del café del mundo (ICO, 20I7). Las plantaciones de café certificado en este continente en promedio tienen un área de o,8ha (UTZ, 2015; Fairtrade International, 2015). En África oriental predomina la especie Arábica, mientras en la región occidental y central lo hace la Robusta, aunque en las zonas altas de esta última también hay cultivos de la especie Arábica (Panhuysen y Pierrot, 2014).

Los países africanos en que se han desarrollado mayor número de investigaciones publicadas sobre los impactos ambientales de la certificación de plantaciones de café son Etiopia, Costa de Marfil, Kenia y Ghana. En la tabla 3 , se registran los principales impactos ecológicos, sociales y económicos que son los más comunes. 
TABLA 3.

Principales impactos generados por la certificación de plantaciones de café en África

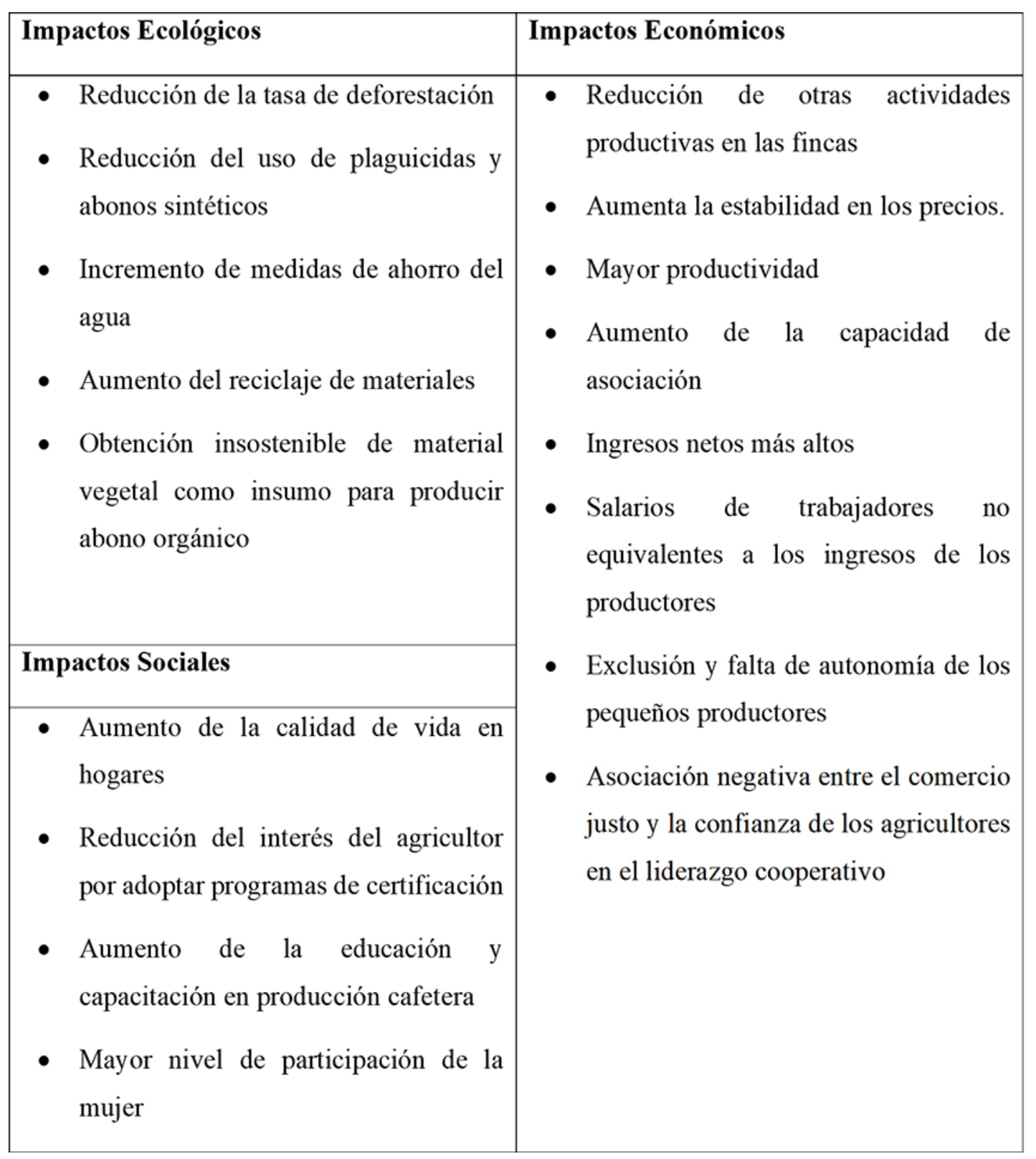

Fuente: elaboración propia

Los impactos ambientales que la certificación cafetera genera son diversos, en algunos casos contrastan dependiendo la región donde se desarrollan. En Etiopía Takahashi y Todo (2013), encontraron que los bosques aledaños a cultivos de café de sombra certificado bajo el programa Rainforest Alliance eran menos propensos a ser deforestados que los bosques que no presentaban cercanía al café o a otros cultivos. Por el contrario, se observó que los bosques cercanos al café sin certificación, no eran menos propensos a ser deforestados que los bosques sin café u otros cultivos cercanos.

El café de sombra también genera otra serie de impactos como la disminución en la temperatura del suelo, el rompimiento en la fuerza del viento y de las precipitaciones, el control en la erosión en pendientes pronunciadas, supresión de malas hierbas, reciclaje y lixiviación de nutrientes, entre otros. Sin embargo, en las regiones donde la lluvia es limitada y las estaciones secas son prolongadas como es el caso de Kenia, Camerún o Tanzania, los árboles 
de sombra pueden afectar negativamente a la productividad debido a una fuerte competencia con el café por la humedad disponible en el suelo (Van der Vossen, 2005).

En el África subsahariana, la aplicación anual de fertilizantes inorgánicos es de sólo $9 \mathrm{~kg} / \mathrm{ha}$ (nutrientes) que frente al promedio mundial de $90 \mathrm{~kg} / \mathrm{ha}$ es muy baja (Dudal, 2002). La caficultura certificada incluye dentro de sus criterios el uso de fertilizantes orgánicos, pero en África son varios los impactos que se derivan de este criterio. Uno de ellos es el cumplimiento del criterio de forma involuntaria, caso de la mayoría de los cafés Arábica en Etiopía, cuyas pequeñas plantaciones se producen sin fertilizantes inorgánicos ni plaguicidas sintéticos debido a la falta de recursos financieros de los productores, por lo tanto, orgánicos por defecto. Aun así, no califican automáticamente como orgánicos (Van der Vossen, 2005).

Resultados de estudios sobre cultivos de campo en África subsahariana, indican que el ciclo de nutrientes en los suelos orgánicos y convencionales son similares. Aunque la materia orgánica es muy importante para mantener la calidad del suelo, los aportes adicionales de fertilizantes inorgánicos en África, siguen siendo necesarios para equilibrar los flujos de nutrientes de las plantas y así lograr niveles de rendimiento adecuados (Vanlauwe et al., 200I; Giller, 2002).

Los residuos de los frutos del café compostado devueltos al campo sólo pueden suministrar 25 a 30\% de estos requerimientos adicionales de nutrientes, por lo que se debe disponer de los medios para adquirir materiales ricos en nutrientes, materia orgánica y abono, para compensar la diferencia y así lograr nutrientes suficientes (Sanchez y Jama, 2002; Van der Vossen, 2005). No obstante, la mayoría de los pequeños productores no cuentan con los recursos para tener acceso regular a cantidades considerables de materia orgánica o de estiércol, lo que los limita o excluye de la posibilidad de certificar sus plantaciones.

Otro impacto que el criterio de uso de fertilizantes orgánicos genera en algunas regiones, es la insostenibilidad a largo plazo de la explotación de biomasa vegetal en descomposición presente en suelos de bosque destinada a servir de nutrientes en el suelo de los cultivos cafeteros, tal y como lo identificaron Metzler-Amieux y Dosso (1998) en la región de Buyenzi, Burundi donde los pequeños caficultores, basaron su abonado exclusivamente en mantillo, recolectado y transportado al suelo de sus plantaciones agotando gradualmente los nutrientes disponibles en ortos suelos.

En el aspecto económico, son varios los impactos que predominan en el continente. Uno de los más importantes se refiere a los ingresos netos más altos para los productores, pero hay poca evidencia de que los productores obtengan grandes aumentos en el precio como resultado de lograr la certificación y en otros casos los precios garantizados no son suficientes para mitigar la inversión de la certificación (Milder y Newson, 2015; Van Rijsbergen et al, 2016). De igual manera se han observado mayores beneficios económicos para los agricultores de mayores propiedades y de mejor posición económica, mientras que los pagos de salarios a los trabajadores no generan beneficios equivalentes (Ruben y Fort, 2012). Mientras que para otros los costos y requisitos de acceso a la certificación, pueden llevar a la exclusión y la falta de autonomía (Guedes et al., 20I4).

En Ruanda, Elder y colaboradores (2013), concluyeron que los incentivos para especializarse proporcionados por algunos certificadores parecen desalentar a los agricultores sobre dirigir su energía hacia prácticas agrícolas con impactos ambientales positivos en el largo plazo, ya que no proporcionan beneficios económicos inmediatos. Sin embargo, Van der Vossen (2005) indica que la certificación reduce simultáneamente otras actividades generadoras de ingresos, ya que las granjas certificadas tienden a especializarse más en la producción de café y pueden descuidar los demás cultivos de alimentos.

La certificación de comercio justo en algunas regiones de Uganda, aumenta el nivel de vida de los hogares en un 30\%, reduce la prevalencia, el nivel de la pobreza (Chiputwa et al., 2015) y facilita el acceso a la capacitación mejorando de la capacidad de aumentar la calidad del café. Sin embargo, se ha evidenciado una asociación negativa entre el comercio justo y la confianza de los agricultores en el liderazgo cooperativo. Mientras se percibe un mayor nivel de participación de las mujeres en las actividades de producción y comercialización (Elder et al., 20Iz). 
Becchetti y Costantino (2008) reportan que los agricultores certificados con el programa de comercio justo Fairtrade en Kenia, presentan mayor éxito en la diversificación de su producción, por lo que se presentaron mejoras en términos de consumo de alimentos en el hogar y la calidad de su dieta. Así mismo se determinó que la mortalidad infantil disminuyó significativamente luego de la certificación y producción bajo este programa.

\section{México y Centroamérica}

El II,3\% del café que se consume en el mundo se produce en Centroamérica y México, incluyendo algunas islas del caribe. Los principales productores de café en Centroamérica son Honduras y Guatemala, seguido por México, Nicaragua y Costa Rica (Donovan y Poole, 20I4; ICO, 2017). El área de las plantaciones de café certificado en esta región, promedian las 2,6ha y 2,oha en el caso de las islas del caribe (UTZ, 20I5; Fairtrade International, 2015), en las cuales predomina la especie Arábica.

México, Costa Rica y Nicaragua son los países en los que se han desarrollado un mayor número de estudios referidos al impacto ambiental de la certificación cafetera. A continuación, en la tabla 4 se exponen los principales impactos generados por la adopción de programas de certificación en fincas cafeteras de Centroamérica.

TABLA 4.

Principales impactos generados por la certificación de plantaciones de café en Centroamérica

\begin{tabular}{|c|c|}
\hline Impactos Ecológicos & Impactos Económicos \\
\hline $\begin{array}{l}\text { - Reducción de la tasa de deforestación } \\
\text { - Incrementos en tasas de deforestación } \\
\text { - Mayor biodiversidad } \\
\text { - Incremento de medidas de ahorro del } \\
\text { agua } \\
\text { - Mayores tasas de supervivencia de } \\
\text { aves migratorias }\end{array}$ & $\begin{array}{l}\text { - Incremento al acceso al crédito } \\
\text { - Modernización del sistema de } \\
\text { producción } \\
\text { - Incremento del costo de los abonos } \\
\text { orgánicos } \\
\text { - Reducción de los rendimientos a causa } \\
\text { - } \text { del uso de fertilizantes orgánicos } \\
\text { - Ampresos netos más altos } \\
\text { - } \text { - Aumentificación diferencia entre precios por } \\
\text { - Ingresos netos más altos }\end{array}$ \\
\hline
\end{tabular}

Fuente: elaboración propia 
Los impactos ambientales generados por la certificación en Centroamérica, presentan contrastes dependiendo de la región e incluso dentro de un mismo país. Barham y Weber (20I2) indican que en México las mejoras significativas en la productividad de las plantaciones de café existentes evidencian la tendencia a que con el tiempo, se reduce la presión en el margen extensivo de las tierras boscosas. No obstante, en México también se reporta un amplio margen de deforestación para establecer nuevas plantaciones de café atribuidas a suplir el bajo rendimiento de los cultivos certificados asociado al uso de fertilizantes orgánicos que al igual que ocurre en Nicaragua, incrementan su costo a causa de su alta demanda, ya que la generación de materia orgánica en las fincas es insuficiente (Beuchelt y Zeller, 20II; Valkila, 2009).

Otro factor incidente en los impactos ecológicos sobre los bosques en las regiones cafeteras fue analizado por Philpott et al. (2007) en México y Costa Rica, observando que las primas en los precios por café de sombrío y otras certificaciones, que sólo incluyen las relaciones ecológicas entre las características del hábitat y la biodiversidad, inducen a los caficultores a plantar en el sotobosque del bosque nativo. Por otro lado, no existen evidencias de que en las áreas recientemente deforestadas no se esté plantando café orgánico, poniendo en entre dicho los principios de la certificación (Jurjonas et al., 20I6).

Estudios realizados en El Salvador y México, comparan fincas bajo diferentes programas de certificación con fincas no certificadas en términos biodiversidad encontrando que en las certificadas es mayor el número de especies de mamíferos, artrópodos y especies arbóreas, así como especies de aves migratorias, pero sin diferencias significativas entre programas de certificación. Por otro lado, si se comparan estas fincas con el bosque nativo aledaño, este conserva mayor riqueza en términos de diversidad biológica (Philpott et al., 2007; Komar, 20I2; Milder y Newson, 2015).

En Nicaragua Donovan y Poole (20I4), indican que el incremento al acceso del crédito dirigido a la expansión de la producción cafetera certificada que ha sido impulsada por el ingreso a los mercados para el café de comercio justo y el café orgánico, contribuye significativamente a la reducción de la pobreza, para incrementar el acceso de activos que permitan modernizar el sistema de producción y actualizar los conocimientos al igual que las habilidades para la producción de café. No obstante, este impacto es más persistente en los grandes productores, dado que reciben créditos a largo plazo, mientras que a pequeña escala las probabilidades de tener acceso al crédito de varios años es limitada, porque estos han demostrado menor capacidad de reinvertir las ganancias de los precios del café o las mejoras de otros créditos a la acumulación del capital físico para la producción en las explotaciones agrícolas.

Cuando los precios de mercado son bajos, los precios para el café certificado en comercio justo y orgánico son sustancialmente más altos que sus contrapartes no certificados (Ponte, 2004; Calo y Wise, 2005). Sin embargo, en algunas regiones de México estos precios más altos no eran suficientes como para satisfacer los costes de producción, por lo que los agricultores dependen luego de los subsidios del gobierno para pagar sus cuentas.

Por su parte Barham et al. (20II), indican que la diferencia en el ingreso de efectivo neto por hectárea entre los hogares certificados con comercio justo, orgánico y convencional en México, proviene principalmente de las diferencias en los rendimientos y no de los precios, demostrando que la certificación en comercio justo y orgánico no proporciona un camino potencial hacia la solución de la pobreza rural persistente. De acuerdo con Katlyn et al. (2013), en El Salvador los pequeños caficultores pasan períodos de hambre debido a que no diversifican su producción, ya que invierten su tiempo y energía exclusivamente a la producción de café, poniendo en riesgo su seguridad alimentaria.

Un estudio comparativo entre productores de café certificado (FairTrade y orgánico) y no certificado de México, Nicaragua, El Salvador y Guatemala determinó que la certificación incrementa los precios del café y por tanto los ingresos, pero que no se observan efectos significativos sobre los medios básicos de subsistencia como salud y educación (Mendez et al., 2010; Grabs et al., 2016). 


\section{Suramérica}

La producción cafetera suramericana excede el $45 \%$ del café del planeta e incluye como sus principales productores a Brasil y Colombia. En menor proporción a otros países andinos como Perú, Ecuador, Bolivia y Venezuela (ICO, 2017). La especie que predomina en las plantaciones suramericanas es la Arábica, sin embargo, en Brasil también incluye la especie Robusta en los cultivos de su territorio con menor altitud (Panhuysen y Pierrot, 20I4). En esta región las áreas de las plantaciones certificadas promedian 3, iha y totalizan las mayores áreas de los principales programas de certificación (UTZ, 2015; Fairtrade International, 2015; Milder y Newson, 2015).

El mayor número de investigaciones que evalúan los impactos de la certificación cafetera en la región, tienen como área de estudio el territorio colombiano y brasilero. Aunque también hay diversos estudios en Perú y Ecuador. En la tabla 5 se exponen los principales impactos ambientales estudiados en esta región.

TABLA 5 .

Principales impactos generados por la certificación de plantaciones de café en Suramérica

\begin{tabular}{|c|c|}
\hline Impactos Ecológicos & Impactos Económicos \\
\hline $\begin{array}{l}\text { - Reducción de la tasa de deforestación } \\
\text { - } \text { Reducción del uso de plaguicidas } \\
\text { - } \text { riberas de los ríos } \\
\text { - Mayor biodiversidad } \\
\text { - Incremento de medidas de ahorro y } \\
\text { calidad del agua } \\
\text { - Mayor protección a los ecosistemas } \\
\text { aledaños a la plantación }\end{array}$ & $\begin{array}{l}\text { - Mayor productividad } \\
\text { - Ingresos netos más altos } \\
\text { - Aumenta la estabilidad en los precios } \\
\text { - Mayor acceso a la certificación } \\
\text { - } \text { mediante grupos asociativos } \\
\text { - Incremento al acceso al crédito } \\
\text { certificación, por falta de beneficio } \\
\text { inmediato } \\
\text { - Modernización del sistema de } \\
\text { producción } \\
\text { - Aumento en costos de fertilizantes } \\
\text { insuficientes }\end{array}$ \\
\hline
\end{tabular}

Fuente: elaboración propia 
La cobertura arbórea en las plantaciones de café permite una mayor disponibilidad de material orgánico, posibilitando el reciclaje de nutrientes que de otro modo no estarían disponibles para el café. También faculta la diversificación en la producción, así como los ingresos, mejorando la calidad de la copa de la planta de café, particularmente en zonas de café ecológicamente sub-óptimas por sus altas temperaturas. Por otro lado, también genera algunos efectos adversos como el daño a los cafetos por la caída de ramas de los árboles de sombra, costos adicionales de mano de obra para la poda regular de árboles cuando hay sombreado excesivo y competencia por el recurso hídrico en regiones con períodos de baja precipitación, entre otras (Van der Vossen, 2005).

En Colombia, el área de bosque denso en zonas cafeteras ha aumentado considerablemente al igual que la cobertura de arbórea en las parcelas certificadas, más que en las no certificadas (Rueda et al., 2015). También muestran zonas de ribera más sanas, con niveles más altos de macroinvertebrados acuáticos sensibles a la contaminación y mayor riqueza de especies de artrópodos en el suelo (Hughell y Newsom, 2013). Se observó la disminución en el uso de plaguicidas, uso de fertilizantes orgánicos y disminución de las aguas residuales (Ibanez y Blackman, 2016).

Según Hardt et al. (2015), la certificación produjo cambios sutiles en la evolución y disponibilidad de hábitat al igual que la conectividad del paisaje en fincas cafeteras brasileras, evaluadas luego de nueve años de certificación bajo el sello Rainforest Alliance, encontrando que esta reduce las tasas de deforestación y mejora la conservación, así como la conectividad de la vegetación nativa local. Sin embargo, Waldron et al. (2012), observó que las medidas para aumentar la biodiversidad en paisajes agrícolas a menudo reducen el rendimiento o aumentan los costos de producción, lo cual generalmente desincentiva a los agricultores para adoptar prácticas amigables con la biodiversidad.

Estudios comparativos realizados en fincas cafeteras certificadas Fairtrade y fincas sin certificación del Perú, concluyen que en términos de ingresos familiares, no hay efecto significativo de la participación en este programa, presuntamente afectado por el aumento de los costos de mano de obra, insumos y la reducción de los ingresos derivados de otras actividades productivas. Así mismo, se evidencia la reducida diferencia de precios del café certificado respecto al no certificado. Sin embargo, se evidencia mayor bienestar general en términos económicos, infraestructura del hogar y de producción en un efecto muy reducido en aspectos como la salud, educación y migración (Ruben, 2012; Barham y Weber, 2012).

Los elevados costos de insumos como el abono orgánico principalmente, afectan directamente los ingresos de los caficultores de la región. Barham y Weber (2012), observaron en Perú, que la producción de materia orgánica para producir abono es insuficiente e incluso, abonos desarrollados a base de guano y otros materiales carecen de un equilibrio óptimo de nutrientes, reduciendo la productividad de la plantación e incrementando los gastos por costos de transporte de fertilizante orgánico.

En Brasil, Guedes et al. (2014) evaluó el acceso de los caficultores a los programas de certificación, encontrando que para los grupos ha aumentado el acceso a los pequeños y medianos productores, debido a los bajos costos, pero con igual exigencia de las auditorías. También observó que a pesar de la accesibilidad que ofrece la certificación mediante grupos asociativos, los productores más marginados todavía no pueden adoptar programas de certificación. En Colombia se observa que al excluirse de la certificación a los productores marginados y sin recursos, estos presentan la tendencia de convertirse paulatinamente en jornaleros de los grandes o medianos productores, abandonando la producción en sus fincas (Guhl, 2009; Grabs et al., 2016).

Los agricultores afiliados a cooperativas de café certificado en buenas prácticas de gestión sostenible de la tierra a menudo tienen más acceso al crédito (Méndez et al., 20I0). Aunque Ibanez y Blackman, (20I6) luego de estudios realizados en Colombia, sugieren que los beneficios económicos de la certificación orgánica son limitados e indican que los costos de los insumos y el bajo rendimiento son los efectos negativos predominantes de la certificación.

Desde el punto de vista social, la certificación impacta positivamente, mejorando las condiciones laborales de los trabajadores de la finca, incrementa el uso de equipos de seguridad y protección personal (Guhl, 2009). 
Sin embargo, estudios realizados por Manrique y Gracia (2014), manifiestan que las condiciones de seguridad alimentaria e inocuidad en fincas productoras no se asocian significativamente con la certificación de los cultivos.

\section{Discusión}

Luego de expuestos los impactos específicos que con mayor incidencia se presentan en los diferentes territorios productores de café, también es importante agregar los impactos coincidentes en la producción cafetera certificada global.

Los impactos ecológicos que la certificación potencializa, hacen referencia a la protección y conservación de la diversidad vegetal y animal que dentro de los sistemas de café con sombrío proporcionan un control de plagas, incrementan la polinización, conservación de especies, captura de carbono y el control de la erosión. Sin embargo, el beneficio potencial económico de estos servicios a menudo pasa desapercibido para los agricultores, ya que los productores con poca frecuencia son recompensados directamente por estos servicios, aunque las fincas de café de sombra con un conjunto diverso de árboles a menudo proporcionan otras fuentes de ingresos para los agricultores, entre los que se incluyen ingresos adicionales de madera y productos no maderables de los árboles de sombra (Jha et al., 20II).

La certificación incluye importantes garantías de beneficios para la conservación de la biodiversidad y la evidencia de la conservación a escala local es importante, pero hay una necesidad de considerar mejor el papel dominante de los procesos a escala de paisaje en el mantenimiento de la biodiversidad y no la fragmentación que aún se presenta. Así mismo, la comprensión de los beneficios relativos a los factores económicos y ecológicos, puede ser importante para la consecución de los sistemas agrícolas sostenibles que contribuyen a los medios de vida de los agricultores y la protección del paisaje cafetero.

Existen altas cantidades de café con doble certificación (orgánico y de comercio justo o BirdFriends/orgánico), algunos argumentan que un esfuerzo coordinado para la incorporación de la triple certificación y la creación de las 'super etiquetas' podría cumplir mejor los objetivos generales de sostenibilidad (Philpott y Dietsch 2003; Ponte, 2004) y por lo tanto contribuir mejor a esfuerzos de conservación. Aunque el principal objetivo del caficultor para la múltiple certificación se centra en tener diversas opciones y así vender al mejor precio según el sello que le otorgue mejor pago en el mercado.

Por su parte, los impactos socioeconómicos que las fincas cafeteras generan por su actividad bajo los principios de los diferentes sellos son menos claros. Debido a los diferentes sistemas económicos en que se desarrolla la producción cafetera global, los impactos positivos principalmente se les atribuyen a factores como la confianza en el liderazgo, la organización democrática interna y la crucial participación de las mujeres (Van Rijsbergen et al., 2016), pero no son comunes en todo el mundo. Entre los impactos de mayor predominancia se encuentra la disminución a la exposición de agentes químicos por parte de los trabajadores, tanto por su uso restringido como por las condiciones de seguridad y protección personal con que se manipulan.

Muchos autores exponen el impacto de beneficio económico en la población cafetera a causa de las denominadas primas que la certificación otorga al valor del café, pero este impacto es afectado por diversos factores propios de cada país e incluso de cada región dentro de un mismo país (Ibanez y Blackman, 20I6; Vellema et al., 20I5). Estos factores hacen referencia principalmente al derecho de propiedad del caficultor, al tipo de asociaciones productoras que se desarrollan y a la oscilación de los precios en el mercado internacional. Se hace evidente, que en las regiones donde predomina el cultivo del grano, se observa un desarrollo amplio en materia de vías secundarias y terciarias. Los gobiernos y asociaciones generalmente impulsan programas para tecnificar la producción, lo que incrementa 
las obras civiles locales e indirectamente incrementa la cobertura educativa y los servicios prestadores de salud para estas comunidades.

Finalmente, una amenaza latente de la certificación cafetera y de otros productos, considerada por Guhl (2009), consiste en que a pesar de que la certificación puede fortalecer los vínculos comerciales entre la producción local y los consumidores internacionales, se corre el riesgo de establecer más vínculos de explotación y solidificación de la dependencia entre el norte y el sur.

\section{Conclusiones}

Se puede observar que el impacto de mayor predominancia causado por la certificación en los principales países productores de café en el mundo, es el incremento de los ingresos netos que viene siendo finalmente lo que motiva a los caficultores a adoptar este tipo de programas en sus fincas, ya que incrementa su poder adquisitivo y acceso a los aspectos que se supone mejoran su calidad de vida. No obstante, un importante grupo de estudios en los diferentes continentes productores indican que las diferencias de estos ingresos comparados con los de los productores no certificados no es significativa, lo que conduce a dudar de la eficiencia de este mecanismo para reducir la pobreza en sector rural.

Por otro lado, los programas de certificación han demostrado su impacto positivo a los agroecosistemas en términos de reducción del uso de agua en los procesos y por ende, reducción del agua contaminada. Así mismo, es evidente en la mayoría de estudios que evalúan aspectos ecológicos resaltan la disminución en el uso de agroquímicos, pero también se presume que esta práctica conlleva a otros impactos generados por el afán de suplir la función de estas sustancias, lo cual requiere ser investigado más a fondo y mediante métodos más directos.

Finalmente se hace evidente que los efectos generados por la adopción de programas de certificación en las fincas cafeteras, presentan contrastes entre países vecinos e incluso entre regiones cafeteras de un mismo país, lo que sustenta la necesidad de continuar evaluando dichos impactos, ya que estos parecen básicamente generarse de acuerdo a las condiciones geográficas y a la organización social de las comunidades productoras.

\section{Referencias}

Arnould, E., A. Plastina, y D. Ball. (2009). Does Fair Trade deliver on its core value proposition? Effects on income, educational attainment, and health in three countries. Journal of Public Policy and Marketing, 28(2), I86-20I. ht tps://doi.org/I0.1509/jppm.28.2.186

Auld, G. (2010). Assessing Certification as Governance: Effects and Broader Consequences for Coffee. The Journal of Environment \&- Development. 19(2) 215-24I. https://doi.org/10.1177/1070496510368506

Auriol, E., y Schilizzi, S. G. M. (2015). Quality signaling through certification in developing countries. Journal of Development Economics, II6, 105-I2I. https://doi.org/10.1016/j.jdevec0.2015.03.007

Baland, J. M., y Duprez, C. (2009). Are labels effective against child labor? Journal of Public Economics, 93(II-I2), II25-I130. https://doi.org/I0.I0I6/j.jpubeco.2009.08.002

Barham, B. L., Callenes, M., Gitter, S., Lewis, J., y Weber, J. (20II). Fair Trade/Organic Coffee, Rural Livelihoods, and the "Agrarian Question": Southern Mexican Coffee Families in Transition. World Development, 39(I), 134-I45. https://doi.org/10.1016/j.worlddev.2010.08.005

Barham, B. L., y Weber, J. G. (2012). The Economic Sustainability of Certified Coffee: Recent Evidence from Mexico and Peru. World Development,40(6), I269-I279. https://doi.org/I0.1016/j.worlddev.2011.11.005 
Becchetti, L., y Costantino, M. (2008). Fair Trade on marginalised producers: An impact analysis on Kenyan farmers. World Development, 36(5), 823-842. https://doi.org/ı0.1016/j.worlddev.2007.05.007

Beuchelt, T. D., y Zeller, M. (20II). Profits and poverty: Certification's troubled link for Nicaragua's organic and fairtrade coffee producers. Ecological Economics, 70(7), 1316-1324. https://doi.org/10.1016/j.ecolecon.2011.01.005

Blackman, A., y Naranjo, M. A. (20I2). Does eco-certification have environmental benefits? Organic coffee in Costa Rica. Ecological Economics, 83, 58-66.: https://doi.org/10.10I6/j.ecolecon.2012.08.00I

Blackman, A., y Rivera, J. (2010). The Evidence Base for Environmental and Socioeconomic Impacts of "Sustainable" Certification. Discussion Paper. Resources for the Future.

Bolwig, S., P. Gibson, y S. Jones. 2009. The economics of smallholder organic contract farming in tropical Africa. World Development, 37(6), 1094-104. https://doi.org/10.1016/j.worlddev.2008.09.012

Calo, M., y Wise, T. A. (2005). Revaluing Peasant Coffee Production: Organic and fair trade Markets in Mexico. Global Development and Environment Institute.

Chi, T. Chien, L., y Chen, S. (2015). Impact of Certification System on Smallhold Coffee Farms' Income Distribution in Vietnam. Asian Journal of Agriculture and Rural Development, 5(5), 137-149. https://doi.org/I0.I 8488/journal.1005/2015.5.5/1005.5.116.123

Chiputwa, B., Spielman, D. J., y Qaim, M. (2015). Food Standards, Certification, and Poverty among Coffee Farmers in Uganda. World Development, 66, 400-4I2. https://doi.org/10.1016/j.worlddev.2014.09.006

COSA. (2013). Vietnam coffee: A COSA Survey of UTZ Certified Farms. Committee on Sustainability Assessment. https://utz.org/wp-content/uploads/2015/12/cosa_baseline_report_on_utz_certified_coffee_far mers_in_vietnam.pdf

Donovan, J., y Poole, N. (2014). Changing Asset Endowments and Smallholder Participation in Higher Value Markets: Evidence from Certified Coffee Producers in Nicaragua. Food Policy, 44, I-13. https://doi.org/I0.I or6/j.foodpol.2013.09.010

Dudal, R. (2002). Forty years of soil fertility in sub-Saharan Africa. En B. Vanlauwe, J. Diels, N. Sanginga, y R. Merckx (eds.), Integrated Plant Nutrient Management in sub-Sabaran Africa: from Concept to Practice (pp. 7-22). CAB International.

Elder, S. D., Zerriffi, H., y Le Billon, P. (2013). Is Fairtrade Certification Greening Agricultural Practices? An Analysis of Fairtrade Environmental Standards in Rwanda. Journal of Rural Studies, 32, 264-274. https://doi .org/10.1016/j.jrurstud.2013.07.009

FairTrade International. (2016). Scope and Benefits of Fairtrade. Seventh Edition. FairTrade FLO. https://www.fairtr ade.net/library/monitoring-the-scope-and-benefits-of-fairtrade-seventh-edition-2015

Ferraro, P. J., Uchida, T., y Conrad, J. M. (2005). Price Premiums for Eco-friendly Commodities: Are 'Green' Markets the Best Way to Protect Endangered Ecosystems? Environmental and Resource Economics, 32(3), 419-438. https://doi.org/10.1007/s10640-005-7962-6

FIBL y IFOAM. (2016). The World of Organic Agriculture. Statistics y Emerging Trends 2016. Research Institute of Organic Agriculture FiBL - IFOAM Organics International. http://www.organic-world.net/yearbook/yea rbook-20I6.html

Giller, K. E. (2002). Targeting Management of Organic Resources and Mineral Fertilizers : Can we Match Scientists’ Fantasies with Farmers’ Realities? En B. Vanlauwe, N. Sanginga, J. Diels, y R. Merckx (eds.), Integrated Plant Nutrient Management in Sub-Saharan Africa (pp. 155-17I) 
Grabs, J., Kilian, B., Hernández, D. C., y Dietz, T. (2016). Understanding Coffee Certification Dynamics: A Spatial Analysis of Voluntary Sustainability Standard Proliferation. International Food and Agribusiness Management Review, 19(3), 3I-56. https://doi.org/I0.22004/ag.econ.244667

Guedes, L. Gardner, T. McDermott, C., y Lara, K. (20I4). Group certification supports an increase in the diversity of sustainable agriculture network-rainforest alliance certified coffee producers in Brazil. Ecological Economics, 107, 59-64. https://doi.org/10.1016/j.ecolecon.20I4.08.006

Guhl, A. (2009). Café, bosques y certificación agrícola en Aratoca, Santander. Revista de Estudios Sociales, (32), II4-I25. https://doi.org/10.7440/res32.2009.08

Haggar, J., Asigbaase, M., Bonilla, G., Pico, J., y Quilo, A. (2015). Tree diversity on sustainably certified and conventional coffee farms in Central America. Biodiversity and Conservation, 24(5), 1175-1194. https://doi.org/ I0.1007/SI053I-0I4-085I-y

Hardt, E, Borgomeo, E, dos Santos, R, Pinto, L, Metzger, J., y Sparovek, G. (2015). Does certification improve biodiversity conservation in Brazilian coffee farms?. Forest Ecology and Management, 357, I8I-194. https://doi. org/10.1016/j.foreco.2015.08.021

Higgins, J. y Green, S. (20II). Cochrane handbook for systematic reviews of interventions (version 5.I.0). The Cochrane Collaboration. www.cochrane-handbook.org.

Hughell, D, y Newsom, D. (2013). Impacts of Rainforest Alliance certification on coffee farms in Colombia. Rainforest Alliance.

Ibanez, M., y Blackman, A. (20I6) Is Eco-Certification a Win-Win for Developing Country Agriculture? Organic Coffee Certification in Colombia. World Development, 82, I4-27. https://doi.org/ı0.1016/j.worlddev.2016.01.004

ICO. (2017). Total Production by all Exporting Countries of Coffee Trade Data. International Coffee Organization. htt p://www.ico.org/prices/po-production.pdf

Jaffee, D. (2008). Better, but not great: the social and environmental benefits and limitations of fair trade for indigenous coffee producers in Oaxaca, Mexico. En R. Ruben (ed.), The Impact of Fair Trade. (pp. 196-222). Wageningen Academic Publishers, The Netherlands.

Jha, S., Bacon, C., Philpott, S., Rice, R., Méndez, V., y Läderach, P. (20II). A Review of Ecosystem Services, Farmer Livelihoods, and Value Chains in Shade Coffee Agroecosystems. En Integrating Agriculture, Conservation and Ecotourism: Examples from the Field (Vol. I) (pp. I4I-208). Springer Netherlands. https://doi.org/10.1007/978-9 4-007-1309-3_4

Jurjonas, M., Crossman, K., Solomon, J., y Lopez, W. (2016). Potential Links Between Certified Organic Coffee and Deforestation in a Protected Area in Chiapas, Mexico. World Development, 78, I3-2I. https://doi.org/10.I oI6/j.worlddev.2015.10.030

Katlyn S.M., Méndez, V.E., y Olson, M.B. (2013). 'Los meses flacos’: seasonal food insecurity in a Salvadoran organic coffee cooperative. The Journal of Peasant Studies, 40(2), 423-446. https://doi.org/10.1080/03066150.20 13.777708

Kessler, M., Hertel, D., Jungkunst, H. F., Kluge, J., Abrahamczyk, S., Bos, M., Buchori, D., Gerold, G., Gradstein, S. R., Köhler, S., Leuschner, C., Moser, G., Pitopang, R., Saleh, S., Schulze, C. H., Sporn, S. G., SteffanDewenter, I., Tjitrosoedirdjo, S. S., y Tscharntke, T. (2012). Can joint carbon and biodiversity management in tropical agroforestry landscapes be optimized? Plos One 7(10), e47192. https://doi.org/10.1371/journal.pon e. 0047192

Komar, O. (2012). Are Rainforest Alliance certified coffee plantations bird-friendly? SalvaNatura Fundacion Ecologica, San Salvador. 
Krasnozhon, L., Simpson, D., y Block, W. (2015). Fair Trade: Its Real Impact on the Working Poor. The Review of Social and Economic, I(2), 6-28. http://www.rebe.rau.ro/RePEc/rau/rseijr/SPI5/RSEI-SPI5-AI.pdf

Lyngbaek, A., R. Muschler, y F. Sinclair. (200I). Productivity and profitability of multistrata organic versus conventional coffee farms in Costa Rica. Agrofestry Systems, 53(2), 205-213. https://doi.org/10.1023/A:101333 2722014

Manrique, O., y Gracia, J. (2014). Seguridad e Inocuidad Alimentaria en Hogares de Jornaleros de Fincas Cafeteras con y sin Certificación del Suroeste de Antioquia - Colombia. Vitae, $2 I(\mathrm{I}), 20-29$. https://revistas.udea.edu. co/index.php/vitae/article/view/15767

Martínez-Sánchez, J. C. (2008). The Role of Organic Production in Biodiversity Conservation in Shade Coffee Plantations (Publication No.). [Ph.D. dissertation, University of Washington]. http://bio-nica.info/Biblioteca/Martin ezSanchez2008TesisCafe.pdf

Martínez-Torres, M. E. (2008). The benefits and sustainability of organic farming by peasant coffee farmers in Chiapas, Mexico. En C. Bacon, V. Méndez, S. Gliessman, D. Goodman, J. Fox, (eds.), Confronting the Coffee Crisis: Fair Trade, Sustainable Liveliboods, and Ecosystems in Mexico and Central America (pp. 99-I26). MIT Press.

Mendez, V., Bacon, C. Olson, M. Petchers, S. Herrador, D. Carranza, C., Trujillo, L., Guadarrama-Zugasti; C., Cordón, A., y Mendoza, A. (2010). Effects of Fair Trade and organic certifications on small-scale coffee farmer households in Central America and Mexico. Renewable Agriculture and Food Systems, 25(3), 236-25I. https://d oi.org/10.1017/S1742170510000268

Méndez, V. E., Bacon, C. M., Olson, M., Morris, K. S., y Shattuck, A. (2010). Agrobiodiversity and shade coffee smallholder livelihoods: a review and synthesis of ten years of research in Central America. The Professional Geographer, 62(3), 357-376. https://doi.org/10.1080/00330124.2010.483638

Metzler-Amieux, V., y Dosso, M. (1998). Conditions de durabilite d'un système agraire caféicole: bilan des transferts de matìere organique à l' èchelle de la colline, au Buyenzi (Burundi). Cabiers Agricultures, 7(4), 27I-279. https://revues.cirad.fr/index.php/cahiers-agricultures/article/view/30097

Meyfroidt, P., Vu, T. P., y Hoang, V. A. (2013). Trajectories of deforestation, coffee expansion and displacement of shifting cultivation in the Central Highlands of Vietnam. Global Environmental Change, 23(5), II87-I198. https ://doi.org/10.1016/j.gloenvcha.2013.04.005

Milder, J., Hart, Abigail K., Dobie, Philip, Minai, Joshua, y Zaleski, Christi. (2014). Integrated Landscape Initiatives for African Agriculture, Development, and Conservation: A Region-Wide Assessment. World Development, 54, 68-80. https://doi.org/10.1016/j.worlddev.2013.07.006

Milder, J., y Newsen, D. (2015). Evaluating the Effects of the SAN/Rainforest Alliance Certification System on Farms, People, and the Environment. SAN/Rainforest Alliance Impacts Report. https://www.rainforest-alliance.org/s ites/default/files/2016-08/SAN_RA_Impacts_Report.pdf

Neilson, J. (2008). Global Private Regulation and Value-Chain Restructuring in Indonesian Smallholder Coffee Systems. World Development, 36(9), I607-1622. https://doi.org/10.1016/j.worlddev.2007.09.005

Panhuysen, S., y Pierrot, J. (2014). Coffee Barometer. IUCN - Hivos - WWF-Oxfam. https://www.preventionw eb.net/files/38295_coffeebarometer2014reporto.pdf

Philpott, S. M., Bichier, P., Rice, R., y Greenberg, R. (2007). Field-Testing Ecological and Economic Benefits of Coffee Certification Programs. Conservation Biology, 2I(4), 975-985. https://doi.org/10.IIII/j.1523-1739.2007.00 728.x

Ponte, S. (2004, I.. de mayo). Standards and Sustainability in the Coffee Sector: A Global Value Chain Approach. In United Nations Conference on Trade and Development and the International Institute for Sustainable 
Development Manitoba. https://www.researchgate.net/publication/264713468_Standards_and_Sustainabilit y_in_the_Coffee_Sector_A_Global_Value_Chain_Approach

Raynolds, L., Murray, D., y Heller, A. (2007). Regulating sustainability in the coffee sector: A comparative analysis of third-party environmental and social certification initiatives. Agriculture and Human Values, 24, I47-I63. ht tps://doi.org/10.1007/sio460-006-9047-8

Rivera, J., de Leon, P., y Koerber, C. (2006). Is greener whiter yet? The Sustainable Slopes Program after five years. Policy Studies Journal, 34(2), 195-224. https://doi.org/10.IIII/j.154I-0072.2006.00166.x

Ruben, R., y Fort, R. (2012). The Impact of Fair Trade Certification for Coffee Farmers in Peru. World Development,40(3), 570-582. https://doi.org/10.1016/j.worlddev.2011.07.030

Rueda, X., Thomas, N., y Lambin, E. (2015). Eco-certification and coffee cultivation enhance tree cover and forest connectivity in the Colombian coffee landscapes. Regional Environmental Change, I5(I), 25-33. https://doi.org /10.1007/SIOII3-014-0607-y

Sáenz, F., y Zúñiga, G. (2008). Assessment of the effect of Fair Trade on smallholder producers in Costa Rica: A comparative study in the coffee sector. En R. Ruben (ed.), The impact of Fair Trade. Wageningen Academic Publishers.

Sanchez, P. A., y Jama, B. A. (2002). Soil fertility replenishment takes off in East and Southern Africa. En B. Vanlauwe, J. Diels, N. Sanginga y R. Merckx (eds.), Integrated Plant Nutrient Management in sub-Sabaran Africa: from Concept to Practice, (pp. 23-45). CAB International.

Smithsonian Migratory Bird Center. (2017). List of farm Bird Friendly Coffee. https://nationalzoo.si.edu/migra tory-birds/certified-coffee-farms

Takahashi, R., y Todo, Y. (2013). The impact of a shade coffee certification program on forest conservation: A case study from a wild coffee forest in Ethiopia. Journal of Environmental Management,I30, 48-54. https://doi.org/I 0.1016/j.jenvman.2013.08.025

Trimarchi, E. (2015). The environmental, social and economic impact of different certification programmes of coffee in Santander, Colombia. A case study on smallholder producers certified with Organic, Fairtrade and Rainforest Alliance. (Publication No. 393312I) [Masther Thesis, Universiteit Utrecht]. Utrecht Universiteit Repository. https://dspace.library.uu.nl/handle/1874/297329

Tscharntke, T., Clough, Y., Bhagwat, S. A., Buchori, ., Faust, H., Hertel, D., Hölscher, D., Juhrbandt, J., Kessler, M., Perfecto, I., Scherber, C., Schroth, G., Veldkamp, E., y Wanger T. C. (20II). Multifunctional shade-tree management in tropical agroforestry landscapes - a review. Journal of Applied Ecology, 48(3), 619-629. https:// doi.org/ I0.11II/j.1365-2664.2010.01939.x

Tscharntke, T., Milder, J. C., Schroth, G., Clough, Y., DeClerck, F., Waldron, A., Rice, R., y Ghazoul, J. (2015). Conserving Biodiversity Through Certification of Tropical Agroforestry Crops at Local and Landscape Scales. Conservation Letters, 8(I), I4-23. https://doi.org/I0.IIII/conl.I2IIO

UTZ. (2015). Facts \& Figures. UTZ Better Farming Better Future. https://www.utz.org/wp-content/uploads/20 16/04/Annual-Report-2015-factsandfigures.pdf

Valkila, J. (2009). Fair Trade organic coffee production in Nicaragua - Sustainable development or a poverty trap? Ecological Economics, 68(12), 3018-3025. https://doi.org/10.1016/j.ecolecon.2009.07.002.

Van der Vossen, H. (2005). A critical analysis of the agronomic and economic sustainability of organic coffee production. Experimental Agriculture, 4I(4), 449-473. https://doi.org/I0.I0I7/S00I4479705002863 
Van Rijsbergen, B, Elbers, W., Ruben, R., y Njuguna, S. (2016). The Ambivalent Impact of Coffee Certification on Farmers' Welfare: A Matched Panel Approach for Cooperatives in Central Kenya. World Development, 77, 277-292. https://doi.org/10.1016/j.worlddev.2015.08.021

Vanlauwe, B., Wendt, J., y Diels, J. (200I). Combined application of organic matter and fertilizer. En G. Tian, F. Ishida y J. D. H. Keatinge (eds.), Sustaining soil fertility in West Africa, (pp. 247-279) Soil Science Society of America Special Publication 58. https://doi.org/ı0.2136/sssaspecpub58.chı2

Vellema, W., Buritica Casanova, A., Gonzalez, C., y D’Haese, M. (2015). The effect of specialty coffee certification on household livelihood strategies and specialisation. Food Policy, 57, 13-25. https://doi.org/ı0.1016/j.foodpo 1.2015 .07 .003

Waldron, A., Justicia, R., Smith, L. E., y Sanchez, M. (2012). Conservation through Chocolate: a win-win for biodiversity and farmers in Ecuador's lowland tropics. Conservation Letters, 5, 213-22I.https://doi.org/I0.IIII/ j.1755-263X.2012.00230.x

Zapata Diomedi, B., y Nauges, C. (2016). Pesticide-handling practices: the case of coffee growers in Papua New Guinea. Australian Journal of Agricultural and Resource Economics, 60, II2-I29. https://doi.org/I0.IIII/I467-8489. I2I06.

\section{Notas}

* Artículo de revisión

CC BY 